(1)

\title{
Associação entre cognição, funcionalidade e fragilidade em idosos da comunidade de Juiz de Fora
}

\author{
Kelly Cristina Atalaia-Silva, ${ }^{1 *}$ Lilian Atalaia-Silva, ${ }^{2}$ Camila A. Faria ${ }^{3}$
}

\section{Resumo}

Introdução: Vários são os estudos que apontam uma relação entre a síndrome da fragilidade e o desempenho cognitivo, apesar do fato de a cognição não fazer parte dos critérios que compõem o fenótipo tradicional da fragilidade. Neste sentido, o objetivo deste trabalho foi avaliar a associação entre os desempenhos cognitivo e funcional e a síndrome de fragilidade em idosos da Rede Fibra. Materiais e Métodos: Foi realizado um estudo transversal em uma amostra de idosos da comunidade da cidade de Juiz de Fora (MG). Foram utilizados os instrumentos MEEM, escalas de ABVD’s, AIVD's e AAVD’s, dinamômetro, escala Minnesota de gasto calórico e a escala CES-D. Resultados: A amostra foi composta por 40 sujeitos frágeis $(79,05 \pm 7,03)$ e 384 sujeitos não frágeis $(73,98 \pm 6,69)$. A idade foi colocada no modelo como covariável, uma vez que foi diferente entre os dois grupos. A ANOVA covariada para idade mostrou que todas as variáveis de funcionalidade e cognição global foram estatisticamente significativas: ABVD ( $\mathrm{F}=2,57$; $\mathrm{p}=0,001) ; \operatorname{AIVD}(\mathrm{F}=6,13 ; \mathrm{p}=0,001) ; \operatorname{AAVD}(\mathrm{F}=2,14 ; \mathrm{p}=0,001)$ e $\operatorname{MEEM}(\mathrm{F}=3,29 ; \mathrm{p}=0,001)$. A análise de regressão identificou que a medida de cognição global e as medidas de funcionalidade, juntas, são capazes de explicar $47 \%$ da variabilidade do desfecho fragilidade ( $\mathrm{F}=29,82 ; \mathrm{p}<0,00 ; \mathrm{R}=0,47)$. Discussão: Os idosos portadores da síndrome da fragilidade apresentaram uma associação maior entre o desempenho nas atividades avançadas e instrumentais da vida diária do que no desempenho cognitivo e nas atividades básicas. Futuros trabalhos devem avaliar o impacto que a capacidade funcional e a cognição podem exercer longitudinalmente em idosos frágeis. Conclusões: $\mathrm{O}$ presente estudo identificou que os idosos frágeis apresentaram comprometimento na cognição e na funcionalidade.

Descritores: Idosos; Fragilidade; Funcionalidade; Cognição.

\section{Abstract}

Association between cognition, functionality and frailty among the elderly community from Juiz de Fora

Introduction: Despite of the fact that cognition is not one of the frailty's traditional phenotypic criteria, there are many papers studying the relationship between frailty's syndrome and cognition's performance. In this sense, the objective of this study was to evaluate the association between the cognitive and functional performances in relation to the fragility syndrome in the elderly of the Fibra Network. Materials and Methods: A cross-sectional study was carried out on a sample of elderly people from the city of Juiz de Fora (MG). We used the instruments MEEM, ABVD's, AIVD's and AAVD's scales,
1. Centro de Ciências da Saúde. Universidade Federal do Recôncavo da Bahia. Santo Antônio de Jesus, BA, Brasil.

2. Departamento de Fisioterapia. Centro Universitário Estácio Juiz de Fora. Juiz de Fora, MG, Brasil.

3. Laboratório de Pesquisa em Envelhecimento Humano. Universidade do Estado do Rio de Janeiro. Rio de Janeiro, RJ, Brasil.

*Endereço para correspondência:

Centro de Ciências da Saúde, Universidade Federal do Recôncavo da Bahia.

Avenida Carlos Amaral, 1.015

Santo Antônio de Jesus, BA, Bahia. CEP: 44570-000.

E-mail: kelly.atalaia@ufrb.edu.br

Revista HUPE, Rio de Janeiro, 2018;17(2):57-64

doi: $10.12957 /$ rhupe.2018.40859

Recebido em 07/05/2018. Aprovado em 08/08/2018.

dynamometer, Minnesota caloric expenditure scale, and the CES-D scale. Results: The sample consisted of 40 fragile subjects (79.05 \pm 7.03$)$ and 384 non-fragile subjects (73.98 \pm 6.69). Age was placed in the model as covariate, since it was different between the two groups. The ANOVA covariates for age showed that all variables of functionality and global cognition were statistically significant: $\operatorname{ABVD}(\mathrm{F}=2.57, \mathrm{p}=0.001)$; AIVD ( $\mathrm{F}=6.13, \mathrm{p}=0.001)$; AAVD $(\mathrm{F}=2.14, \mathrm{p}=0.001)$ and MMSE $(\mathrm{F}=3.29, \mathrm{p}=0.001)$. The regression analysis identified that the measure of global cognition and functional measures together are able to explain $47 \%$ of the variability of the outcome fragility ( $\mathrm{F}=29.82 ; \mathrm{p}<0.00 ; \mathrm{R}=0.47$ ). Discussion: Elderly individuals with fragility syndrome had a greater association between performance in the advanced and instrumental activities of daily living than in cognitive performance and basic activities. Future work should assess the impact that functional capacity and cognition can have on the fragile elderly. Conclusions: The present study identified that frail elderly showed cognitive and functional impairment.

Keywords: Elderly; Frailty; Functionality; Cognition.

\section{Resumen}

\section{Relación entre cognición, funcionalidad y fragili-} dad en ancianos que viven en Juiz de Fora

Introducción: Son varios los estudios que avalan una relación entre el síndrome de fragilidad y la cognición, aunque la cogni- 
ción no tenga parte en los criterios tradicionales del fenotipo del síndrome de fragilidad. En esta dirección, este trabajo tuvo como objectivo evaluar la asociación entre cognición, funcionalidad y el síndrome de fragilidad en ancianos de la Red Fibra. Materiales y métodos: Ha sido realizado um estudio transversal en una población de ancianos de la ciudad de Juiz de Fora (MG). Han sido utilizados los instrumentos MEEM, escalas que miden ABVD’s, AIVD’s y AAVD’s, dinamómetro, escala Minnesota y escala CES-D. Resultados: La muestra incluyó 40 participantes frágiles $(79,05 \pm 7,03)$ y 384 participantes no frágiles $(79,05 \pm 7,03)$. Se agregó la edad como covariable, puesto que esta variable fue diferente entre los dos grupos. El análisis de varianza ANOVA covariada para la edad mostró que todas las variables de funcionalidad y cognición global tuvieron significación estadística: $\mathrm{ABVD}(\mathrm{F}=2,57 ; \mathrm{p}=0,001)$; $\mathrm{AVD}(\mathrm{F}=6,13$; $\mathrm{p}=0,001) ; \operatorname{AAVD}(\mathrm{F}=2,14 ; \mathrm{p}=0,001)$ y $\operatorname{MEEM}(\mathrm{F}=3,29 ; \mathrm{p}=0,001)$.

\section{Introdução}

A síndrome da fragilidade tem maior prevalência nas idades mais avançadas e o desempenho cognitivo e funcional dos idosos tende a piorar com o aumento da idade, podendo evoluir para alterações importantes. ${ }^{1,2}$ Alguns estudos mostram a associação entre a síndrome da fragilidade e o desempenho cognitivo e funcional no envelhecimento. ${ }^{3-5,7}$ A síndrome da fragilidade é definida por alterações relacionadas ao envelhecimento, caracterizadas por diminuição de reserva de energia, dificuldade do organismo em responder ao estresse de forma adequada, sarcopenia, desregulação neuroendócrina e disfunção imunológica. ${ }^{8}$ Estas alterações deixam o organismo mais debilitado, com o sistema de defesa pouco eficaz. Frente a eventos adversos, o idoso com fragilidade pode apresentar dificuldade no restabelecimento das funções fisiológicas, gerando consequências negativas como quedas e fraturas, menor ganho com as intervenções terapêuticas, aumento na taxa de hospitalização e de mortalidade. Além disso, há consequências negativas para a independência e qualidade de vida desses indivíduos. ${ }^{8}$ Os primeiros estudos sobre a síndrome da fragilidade a consideravam como perda na autonomia e na capacidade para realizar atividades da vida diária (AVD's). ${ }^{9-12}$

As AVD's são atividades presentes no cotidiano e indivíduos saudáveis as realizam de forma satisfatória. A perda da habilidade de fazer essas tarefas e de resolver problemas cotidianos resulta em necessidade maior de cuidados, perda de autonomia e de qualidade de vida. ${ }^{13}$ As AVD's são classificadas em básicas, instrumentais e avançadas. As atividades básicas (ABVD's) são
El análisis de regresión ha identificado que la cognición y las escalas de funcionalidad, cuando puestas juntas, pueden explicar 47\% de la variabilidad total del síndrome ( $\mathrm{F}=29,82$; $\mathrm{p}<0,00 ; \mathrm{R}=0,47$ ). Discusión: Los ancianos que presentan el síndrome de fragilidad tuvieron una asociación más grande entre las actividades avanzadas e intrumentales de vida diária en relación al rendimiento cognitivo y actividades básicas. Trabajos futuros deben evaluar el impacto que la capacidad funcional y la cognición pueden ejercer a lo largo del tiempo en ancianos frágiles. Conclusiones: El presente trabajo ha identificado que los ancianos frágiles presentaron perjuicio en la cognición y en la funcionalidad.

Palabras clave: Ancianos; Fragilidad; Funcionalidad; Cognición. habilidades comolevantar-se, tomar banho e alimentarse. ${ }^{14}$ As atividades instrumentais (AIVD's) são tarefas como administrar a vida financeira, cumprir os compromissos e fazer uso correto de medicamentos. ${ }^{13}$ As atividades avançadas da vida diária (AAVD's) estão associadas ao engajamento do idoso nos contextos de lazer e trabalho. ${ }^{15}$ Um estudo realizado por Fried e colaboradores mostrou que $6,3 \%$ da população do Cardiovascular Health Study foi identificada como frágil e 45,3\% como portadora de fragilidade intermediária (pré-frágil). ${ }^{8}$ Dos idosos classificados como frágeis, 39\% apresentaram piora nas ABVD’́s, contra 8\% dos nãofrágeis. ${ }^{8}$ Snih e colaboradores investigaram a associação entre o status de fragilidade eincidência de dependência nas AVD's em 1.645 idosos mexicanos-americanos. ${ }^{16}$ No início do estudo, todos eram independentes para AVD's, $50 \%$ não eram frágeis, $45,7 \%$ eram pré-frágeis e 4,3\% eram frágeis. Após 10 anos, a taxa de risco de dependência nas AVD's para pré-frágeis foi de 1,32 e de 2,42 para os indivíduos frágeis, comparados com os não frágeis. Os autores concluíram que o status de fragilidade está associado com um risco aumentado de dependência nas AVD's após 10 anos.

Em 2006, o grupo de pesquisa de Fried apresentou um modelo biológico para a fragilidade, que seria decorrente de mudanças moleculares e variações genéticas. Estas dariam origem a inflamações e à desregulação neuroendócrina, que, em interação, favoreceriam a anorexia, a sarcopenia, a osteopenia, o declínio da função imunológica, problemas hematológicos e problemas no metabolismo da glucose, 
além de deficit cognitivo. ${ }^{17}$ Alguns pesquisadores entendem a síndrome da fragilidade como fator de risco para o declínio da cognição. ${ }^{3-6}$ Samper-Ternent e colaboradores concluíram que a fragilidade é um fator de risco para a diminuição do escore no Miniexame do Estado Mental (MEEM). ${ }^{4,18} \mathrm{Yu}$ e colaboradores, em um estudo longitudinal de 4 anos, identificaram que os idosos não frágeis na linha de base, porém cognitivamente comprometidos, evoluíram para um quadro de fragilidade $(\mathrm{p}<0,01){ }^{19}$ Tyrovolas e colaboradores avaliaram os aspectos multidimensionais da fragilidade, analisando se o deficit cognitivo pode ser considerado com um dos aspectos constituintes da síndrome da fragilidade. ${ }^{20}$ Por meio do estudo multicêntrico COURAGE, os pesquisadores avaliaram idosos espanhóis, finlandeses e poloneses. Através da análise fatorial do índice multifatorial da fragilidade, os autores identificaram que a dimensão cognição deve ser analisada com um fator que contribui para a explicação da síndrome.

No Brasil, Yassuda e colaboradores estudaram 384 idosos da cidade de São Paulo com o objetivo de avaliar a associação entre os critérios diagnósticos de fragilidade de Fried e colaboradores e o desempenho cognitivo. ${ }^{1,5}$ Os resultados mostraram que o status de frágil estava associado ao baixo desempenho cognitivo, mas os autores consideraram a possibilidade de os fenômenos ocorrerem simultaneamente. Em um segundo estudo com a mesma amostra, Macuco e colaboradores mostraram que a fragilidade, junto com idade, educação e renda familiar, influenciou o escore do MEEM. ${ }^{6}$ Faria e colaboradores estudaram 737 indivíduos com 65 anos ou mais residentes no Rio de Janeiro. ${ }^{3} \mathrm{~A}$ idade e a escolaridade foram avaliadas como possíveis modificadoras de efeito da associação entre fragilidade e desempenho cognitivo. Os pesquisadores mostraram que a síndrome da fragilidade prediz o baixo desempenho cognitivo em idosos e que a idade atua como modificadora de efeito nesta associação. Brigola e colaboradores realizaram um estudo de revisão sistemática e identificaram que todos os 19 estudos analisados encontraram associação entre fragilidade e desempenho cognitivo. ${ }^{21}$

Alguns autores entendem que deficit cognitivo no envelhecimento, junto com variáveis sociais, econômicas, demográficas e de saúde, pode ser fator de risco para fragilidade. Raji e colaboradores realizaram uma pesquisa com 942 idosos não frágeis com o objetivo de verificar a associação entre cognição e o risco de se tornar frágil.? Os autores constataram que, após dez anos, aqueles com baixo desempenho cognitivo (escore menor que 21 no MEEM) teriam um risco maior de se tornarem frágeis do que os idosos com desempenho cognitivo com escore maior ou igual a 21 no MEEM.

A partir dos estudos expostos anteriormente, postulamos como objetivo investigar a associação entre os desempenhos cognitivo e funcional em relação à síndrome de fragilidade em idosos da Rede Fibra Juiz de Fora.

\section{Materiais e métodos}

Esta pesquisa foi parte do projeto multicêntrico rede FIBRA Brasil de delineamento transversal e analítico realizado pelo polo UERJ, realizado com os dados da população idosa do município de Juiz de Fora. ${ }^{22}$ A pesquisa respeitou todos os padrões éticos aprovados pela Comissão Nacional deÉtica em Pesquisa do Ministério da Saúde (CONEPE/MS - 313/2008) sob o processo número 555087/2006-9 com o apoio do Conselho Nacional de Desenvolvimento Científico e Tecnológico (CNPq). A coleta de dados ocorreu nos anos 2008-2009, nas residências dos idosos e foi realizada por pesquisadores da Universidade Federal de Juiz de Fora devidamente treinados.

\section{Instrumentos}

Para a avaliação da cognição global foi utilizado:

- Miniexame do estado mental: avalia as funções cognitivas de orientação tempo/espaço, memória episódica, atenção (cálculo), linguagem ecapacidade visuo-construtiva. ${ }^{18,23} \mathrm{O}$ MEEM é composto por questões agrupadas em cinco categorias: orientação temporal (5 pontos) e espacial (5 pontos); registro de três palavras ( 3 pontos) e evocação das três palavras (3 pontos); cálculo (5 pontos); escrita, nomeação de objetos, compreensão de comandos falados e escritos (8 pontos) e cópia de uma figura (1 ponto). $\mathrm{O}$ escore do MEEM pode variar de um mínimo de zero até um total máximo de 30 pontos. A escala é breve, simples, abrangente, e pode ser administrada de 5 a 10 minutos por qualquer profissional treinado da área de saúde. O material é composto por lápis e papel.

A avaliação da funcionalidade foi mensurada através de três questionários de autorrelato, a saber:

- Atividades Básicas de Vida Diária (ABVD):24,25 São as habilidades consideradas básicas, de autocuidado. Nesta escala, o desempenho nas ABVD's é avaliado 


\section{Artigo original}

através dos itens: 1) tomar banho; 2) vestir-se; 3) usar o vaso sanitário; 4) transferência (deitar e levantar da cama e sentar e levantar da cadeira); 5) continência urinária ou fecal e 6) alimentação. Em cada pergunta, o acompanhante deve responder se o idoso é independente, se precisa de ajuda ou se é dependente para aquela atividade.

- Atividades Instrumentais de Vida Diária (AIVD):26 Referem-se à capacidade de gerir compromissos e/ou atividades cotidianas. Esta escala avalia o desempenho do idoso nas seguintes atividades instrumentais: 1) usar o telefone; 2) usar transporte; 3) fazer compras; 4) preparar alimentos; 5) realizar tarefas domésticas; 6) usar medicamentos e 7) administrar despesas financeiras. Em cada pergunta, o acompanhante deve responder se o idoso é independente, se precisa de ajuda ou se é dependente para aquela atividade.

- Atividades Avançadas de Vida Diária (AAVD): ${ }^{27}$ São atividades mais complexas que envolvem independência e participação social, exemplificadas pela capacidade de dirigir sozinho, viajar, realizar trabalho voluntário e participar de grupos como entidades políticas, associações, entre outros.

Os idosos foram classificados como frágeis e não frágeis de acordo com o fenótipo de fragilidade desenvolvido por Fried e colaboradores, composto por cinco características: perda de peso não intencional, lentificação da marcha, baixo nível de força de preensão palmar, baixo gasto quilocalórico e fadiga. ${ }^{1}$ Para o indivíduo ser considerado frágil era necessário apresentar pelo menos três das cinco características mencionadas. Segue a descrição de cada uma abaixo:

- Perda de peso: foi avaliada através de autorrelato. Foram considerados positivos os sujeitos com perda de mais de 4,5 $\mathrm{Kg}$ ou mais de $5 \%$ do peso corporal durante o último ano, de forma não intencional, ou índice de massa corporal menor que $18,5 \mathrm{Kg} /$ $\mathrm{m}^{2}$, conforme sugerido por Fried e colaboradores. ${ }^{1}$

- Lentificação da marcha: usou-se o cronômetro para medir o tempo gasto para caminhar em um percurso de ida e de volta, cada um com 4,5m, em linha reta. Foram considerados positivos os sujeitos do primeiro quintil após ajuste para altura e tempo, conforme sugerido por Fried e colaboradores. ${ }^{1}$

- Baixo nível de força de preensão palmar: medida por meio de dinamômetro manual(JAMAR Modelo J00105 + formulário de registro Jamar) no membro superior dominante, solicitando ao participante que, por três vezes, exercesse a maior força possível. Foram considerados positivos os sujeitos no primeiro quintil, após ajuste do resultado para sexo e índice de massa corporal, conforme sugerido por Fried e colaboradores. ${ }^{1}$

- Baixo gasto quilocalórico: avaliado por meio do Minnesota Leisure Time Activities Questionnaire. ${ }^{28}$ O Minnesota avalia a atividade física realizada pelo sujeito. Ele é constituído por uma lista de atividades físicas claramente descritas. O entrevistador registrou se cada atividade física foi realizada nas duas últimas semanas. Caso a pessoa tenha praticado a atividade física em questão, ela deve responder quantas vezes o fez em cada semana e quanto tempo gastou, em horas e/ou minutos, cada vez que realizou a atividade. O gasto calórico estimado (por minuto) foi obtido por meio deste questionário. $\mathrm{O}$ cálculo considera o peso corporal do indivíduo e o número de equivalentes metabólicos (MET - metabolic equivalents; $1 \mathrm{MET}=$ 0,0175 Kcal x kg-1 x min-1) necessários para realizar a atividade. A quantidade de METs necessárias para cada atividade é conhecida previamente. ${ }^{28} \mathrm{~A}$ fórmula usada para o cálculo do gasto calórico é a seguinte: Gasto energético $(\mathrm{Kcal} / \mathrm{min})=0,0175 \mathrm{Kcal}$ x kg-1 x min-1 x METs x peso corporal (kg). Foram considerados positivos neste item de fragilidade, os sujeitos do primeiro quintil, conforme sugerido por Fried e colaboradores. ${ }^{1}$

- Fadiga: foi avaliada por meio dos itens 7 e 20 da CES-D - Center of Epidemiological Study Center Scale. ${ }^{28}$ Foram considerados positivos os sujeitos que declarararam fadiga em pelo menos uma das perguntas citadas, conforme sugerido por Fried e colaboradores: "(7) Sentiu que teve que fazer esforço para dar conta das suas tarefas habituais?" e "(20) Não conseguiu levar adiante suas coisas?".

\section{Análise estatística}

Primeiramente foi realizado o teste t para verificar a diferença de médias nos quesitos escolaridade, cognição e funcionalidade entre frágeis e não frágeis. A seguir, para maior controle e rigor metodológico, foi realizada ANOVA entre as variáveis de interesse, covariada para idade. Verificou-se a associação entre função cognitiva e funcionalidade através da Correlação de Pearson. Posteriormente foi feita regressão logística, tendo como variáveis independentes "funcionalidade e cognição" e variável dependente "fragilidade", no intuito de investigar o quanto o modelo composto 
pelas variáveis independentes supracitadas explicaria o desfecho fragilidade.

\section{Resultados}

A amostra foi composta por 40 sujeitos frágeis (média de idade de $79,05 \pm 7,03$ ) e 384 não frágeis (média de idade de 73,98 $\pm 6,69$ ). $O$ teste de Levene para igualdade de varianças indicou que a escolaridade não foi significativamente diferente entre os dois grupos $(\mathrm{F}=4,55 ; \mathrm{p}<0,07)$; porém, a idade foi diferente entre os grupos $(\mathrm{F}=0,02, \mathrm{p}<0,00)$. A diferença entre os grupos também foi observada no MEEM, nas ABVD's, nas AIVD's e nas AAVD's (Tabela 1).

A idade foi colocada no modelo como covariável, uma vez que foi diferente entre os dois grupos. A ANOVA covariada para idade mostrou que todas as variáveis de funcionalidade e cognição global foram estatisticamente significativas: $\mathrm{ABVD}(\mathrm{F}=2,57 ; \mathrm{p}=0,001)$; $\operatorname{AIVD}(\mathrm{F}=6,13 ; \mathrm{p}=0,001) ; \operatorname{AAVD}(\mathrm{F}=2,14 ; \mathrm{p}=0,001)$ e MEEM $(\mathrm{F}=3,29 ; \mathrm{p}=0,001)$. Portanto, mesmo retirando uma possível interferência da idade, as variáveis de interesse continuam sendo significativas.

A correlação de Pearson mostrou associação estatisticamente significativa entre cognição global com idade e todas as variáveis de funcionalidade (Tabela 2).

Ao fazer a análise de regressão, tendo como variável dependente a fragilidade (frágil/não frágil), foi identificado que a medida de cognição global e as medidas de funcionalidade, juntas, são capazes de explicar $47 \%$ da variabilidade do desfecho fragilidade $(\mathrm{F}=29,82 ; \mathrm{p}<0,00 ; \mathrm{R}=0,47)$.

Tabela 1. Diferenças entre as variáveis sociodemográficas, cognitivas e funcionais entre idosos frágeis e não frágeis

\begin{tabular}{|c|c|c|c|c|}
\hline Total de anos estudados & Média & Desvio Padrão & $\mathrm{F}$ & $p<$ \\
\hline Frágeis & 4,1 & 3,7 & --- & --- \\
\hline Não frágeis & 5,5 & 4,2 & --- & --- \\
\hline Diferença entre grupos & --- & --- & 4,55 & 0,04 \\
\hline \multicolumn{5}{|l|}{ MEEM } \\
\hline Frágeis & 22,2 & 3,9 & --- & --- \\
\hline Não frágeis & 24,8 & 3,5 & --- & --- \\
\hline Diferença entre grupos & --- & --- & 0,55 & 0,00 \\
\hline \multicolumn{5}{|l|}{ ABVD's } \\
\hline Frágeis & 17,1 & 1,8 & --- & --- \\
\hline Não frágeis & 17,8 & 0,5 & --- & --- \\
\hline Diferença entre grupos & --- & --- & 129,04 & 0,00 \\
\hline \multicolumn{5}{|l|}{ AIVD's } \\
\hline Frágeis & 16,6 & 3,9 & --- & --- \\
\hline Não frágeis & 20,1 & 1,7 & -- & --- \\
\hline Diferença entre grupos & --- & -- & 98,22 & 0,00 \\
\hline \multicolumn{5}{|l|}{ AAVD's } \\
\hline Frágeis & 22,6 & 2,8 & --- & --- \\
\hline Não frágeis & 25,8 & 3,6 & --- & --- \\
\hline Diferença entre grupos & --- & -- & 0,51 & 0,00 \\
\hline
\end{tabular}

MEEM: miniexame do estado mental; ABVD's: atividades básicas de vida diária; AIVD's: atividades instrumentais de vida diária; AAVD's: atividades avançadas de vida diária. 


\section{Artigo original}

Tabela 2. Correlação entre as variáveis $(p=0,00)$

\begin{tabular}{|c|c|c|c|c|c|}
\hline & Idade & ABVD'S & AIVD'S & AAVD'S & MEEM \\
\hline Idade & --- & $-0,22$ & $-0,47$ & $-0,23$ & $-0,33$ \\
\hline ABVD's & --- & --- & 0,59 & 0,15 & 0,25 \\
\hline AIVD's & --- & --- & --- & 0,34 & 0,40 \\
\hline AAVD's & --- & --- & --- & --- & 0,30 \\
\hline MEEM & --- & --- & --- & --- & --- \\
\hline
\end{tabular}

MEEM: miniexame do estado mental; ABVD's: atividades básicas de vida diária; AIVD's: atividades instrumentais de vida diária; AAVD's: atividades avançadas de vida diária.

A fim de verificar qual a contribuição explicativa de cada variável em separado, foram realizadas análises de regressão com cada variável de interesse. A cognição global explicou $21 \%$ do desfecho fragilidade $(\mathrm{F}=18,62$; $\mathrm{p}<0,00 ; \mathrm{R}=0,21$ ), as AAVD's explicaram 25\% ( $\mathrm{F}=28,88$; $\mathrm{p}<0,00 ; \mathrm{R}=0,25)$, as ABVD's, 30\% ( $\mathrm{F}=40,57 ; \mathrm{p}<0,00$; $\mathrm{R}=0,30$ ), e as AIVD's, 46\% ( $\mathrm{F}=111,19 ; \mathrm{p}<0,00 ; \mathrm{R}=0,46$ ).

\section{Discussão}

Os indivíduos considerados frágeis obtiveram um desempenho inferior tanto na cognição global quanto em todos os níveis de funcionalidade. É esperado que idosos com comprometimento do fenótipo de fragilidade, tais como redução de velocidade de marcha, fadiga, perda de peso não intencional, diminuição da força muscular e baixo gasto energético, possuam maior dificuldade nas atividades de vida diária em relação aos idosos saudáveis. ${ }^{1} \mathrm{O}$ trabalho de Snih e colaboradores mostrou que idosos frágeis tinham duas vezes mais chances de ter alguma limitação em qualquer atividade de vida diária do que os não frágeis, além disso, os frágeis tinham menor instrução educacional e um desempenho inferior no MEEM. ${ }^{16}$ Em um estudo longitudinal, foi observado que idosos não frágeis, porém cognitivamente comprometidos, apresentam um alto risco de tornarem-se frágeis em um seguimento de quatro anos quando comparados a idosos cognitivamente saudáveis. ${ }^{19}$

Vella e colaboradores realizaram um estudo de revisão sistemática a fim de identificar o aumento do uso das medidas cognitivas na definição operacional da síndrome da fragilidade. ${ }^{30}$ Os autores identificaram que tem crescido o número de estudos que utilizam a cognição como indicativo de declínio funcional na síndrome da fragilidade. Apesar deste aumento na utilização de indicadores cognitivos, ainda não há uma sistematização operacional e padronizada sobre a avaliação deste construto dentro dos índices que avaliam a fragilidade. Bunce, Batterham e Mackinnon identificaram que o desfecho fragilidade estava associado a baixo desempenho na velocidade de processamento da informação, na fluência verbal e no tempo de reação na linha de base. ${ }^{31}$ Lee e colaboradores avaliaram a associação entre fragilidade e prejuízo cognitivo em relação à mortalidade em um estudo longitudinal de três anos em idosos coreanos..$^{32}$ Os idosos caracterizados como pré-frágeis e frágeis na linha de base tiveram maiores taxas de mortalidade. O comprometimento cognitivo foi associado a um aumento de $30 \%$ na taxa de mortalidade.

Outro fator importante evidenciado no presente estudo foi o fato de as atividades avançadas apresentarem mais associação com as atividades básicas do que com as atividades instrumentais quando analisada a amostra como um todo, sem estratificação entre frágeis e não frágeis. Caberia esperar que as atividades avançadas guardassem mais relação com as atividades instrumentais, uma vez que, pela hierarquia de complexidade das capacidades funcionais, as AAVD's assemelham-se mais com as AIVD's. Njegovan e colaboradores realizaram um estudo longitudinal em idosos da comunidade e identificaram que a redução dos escores em tarefas de avaliação cognitiva apresentou associação com a perda funcional de atividades mais complexas, como ir ao banco ou fazer compras. ${ }^{33}$ Os autores não encontraram associação entre perda cognitiva e declínio nas atividades básicas. Figueiredo e colaboradores observaram em uma amostra de idosos brasileiros que um bom desempenho nas atividades 
instrumentais e avançadas de vida diária estavam associados a melhores índices na avaliação cognitiva. ${ }^{34}$

Em um estudo de revisão sistemática, Paterson e Warburton encontraram que entre idosos de 65 a 85 anos residentes na comunidade, um alto nível de independência e atividade física estava relacionado a um melhor desempenho cognitivo e risco reduzido de desenvolvimento de demência. ${ }^{35}$ Christelis e Dobrescu realizaram um estudo do banco de dados SHARE de 11 países europeus e encontraram que os idosos que possuíam melhores escores nas atividades avançadas de vida diária eram os que apresentavam melhores indicadores de avaliação cognitiva. ${ }^{36} \mathrm{Já}$ em relação à funcionalidade e o estado de humor, ÁvilaFunes e colaboradores identificaram em um estudo longitudinal que a presença de sintomas depressivos em idosos supostamente saudáveis era capaz de predizer a perda das atividades instrumentais de vida diária, mas não predizia a perda nas atividades básicas. ${ }^{37}$ Talvez, pela própria hierarquia de complexidade, os idosos frágeis apresentem perdas mais significativas em atividades avançadas e instrumentais de vida diária pelo fato de ainda não estarem doentes, mas sim, por estarem fragilizados. As atividades básicas parecem estar mais associadas a perdas patológicas focais e incapacitantes, como a progressão de quadros demenciais ou outras patologias que impeçam a realização de atividades de autocuidado. Nesta direção, torna-se importante avaliar as AAVD’s e as AIVD’s em idosos frágeis que estão na comunidade, a fim de que a estimulação e a reabilitação no desempenho destas atividades possam favorecer o adiamento de perdas funcionais mais graves. Cabe levar em consideração, também, a possibilidade, de os idosos apresentarem maior comprometimento físico, representado pelas ABVD's que comprometimentos cognitivos mais pronunciados, identificados pelas AIVD's, como manuseio de dinheiro. Talvez, a fragilidade física tenha mais relação com as AAVD's que as AIVD's, uma vez que as atividades avançadas pressupõem engajamento social.Para que o idoso tenha um engajamento social, é necessário que ele tenha suas ABVD's preservadas. Para verificar a relação entre tais variáveis, é necessário realizar estudos que sejam capazes de separar as diferentes nuances de capacidade funcional primordialmente em atributos físicos e/ou cognitivos, estratificando-as entre as populações de idosos frágeis e não frágeis.

Por outro lado, ao avaliar o desfecho fragilidade, foi identificado, a partir dos resultados obtidos no presente estudo, que os idosos portadores da síndrome da fragilidade apresentaram uma associação maior entre o desempenho nas atividades avançadas e instrumentais da vida diária do que entre o desempenho cognitivo e as atividades básicas. Uma vez que a síndrome da fragilidade está relacionada a um declínio global de reservas de energia, não implicando necessariamente em prejuízo físico ou cognitivo, talvez as atividades avançadas e instrumentais sejam mais potentes para explicar o desfecho fragilidade pelo fato de envolverem atividades que preconizam habilidades mais sofisticadas, indicando perdas mais sutis. As pessoas portadoras da síndrome da fragilidade podem ou não evoluir para patologias que envolvam perdas cognitivas e físicas acentuadas. Sendo assim, sugerimos que futuros trabalhos avaliem o impacto que a capacidade funcional pode exercer longitudinalmente em idosos frágeis.

\section{Conclusões}

O presente estudo identificou que os idosos frágeis apresentaram comprometimento na cognição e na funcionalidade quando comparados a idosos não frágeis. Além disso, o desfecho fragilidade apresentou uma associação maior entre o desempenho nas atividades avançadas e nas atividades instrumentais da vida diária do que entre o desempenho cognitivo e as atividades básicas de vida diária.

\section{Referências}

1. Fried LP, Tangen CM, Walston J, et al. Frailty in older adults: evidence for a phenotype. J Gerontol A Biol Sci Med Sci. 2001;56(3):M146-57

2. Mesulam M. Aging, alzheimer's disease, and dementia/clinical and neurobiological perspectives. In: Mesulam M. Principles of Behavioral and Cognitive Neurology. Oxford: Oxford University Press, 2000.

3. Faria CA, Lourenço RA, Ribeiro PCC, et al. Desempenho cognitivo e fragilidade em idosos clientes de operadora de saúde. Rev. Saúde Pública [Internet]. 2013 Oct;47(5):923-930. Disponível em: http://www.scielo.br/scielo.php?script=sci_ arttext\&pid=S0034-89102013000500923\&lng=en. http://dx.doi. org/10.1590/S0034-8910.2013047004451

4. Samper-Ternent R. Snih S, Raji MA, et al. Relationship between frailty and cognitive decline in older Mexican Americans. J Am Geriatr Soc. 2008;56(10):1845-52.

5. Yassuda MS, Lopes A, Cachioni M, et al. Frailty criteria and cognitive performance are related: data from the FIBRA study in Ermelino Matarazzo, São Paulo, Brazil. J Nutr Health Aging. 2012;16(1):55-61.

6. Macuco CR, Batistoni SST, Lopes A, et al. Mini-Mental State Examination performance in frail, pre-frail, and non-frail community dwelling older adults in Ermelino Matarazzo, São Paulo, Brazil. Int Psychogeriatr. 2012;24(11):1725-31.

7. Raji MA, Snih S, Ostir GV, et al. Cognitive status and future risk of frailty in older Mexican Americans. J Gerontol A Biol Sci Med Sci. 2010;65(11):1228-34. 


\section{Artigo original}

8. Fried LP. Establishing benchmarks for quality care for an aging population: caring for vulnerable older adults. Ann Intern Med. 2003;139(9):784-6.

9. Woodhouse KW, O'Mahony MS. Frailty and ageing. Age ageing. 1997;26(4):245-6.

10. Gillick M. Pinning down frailty. J Gerontol A Med Sci. 2001;56(3):M134-35.

11. Morley JE, Perry HM, Miller DK. Something about frailty. J Gerontol. 2002;57A(11):M698-704.

12. Hogan DB, Macknight C, Bergman H. Models, definitions, and criteria of frailty. Aging Clinical \& Experimental Research. 2003;15(3):2-29.

13. Razani J, Casas R, Wong JT, et al. The Relationship Between Executive Functioning and Activities of Daily Living in Patients With Relatively Mild Dementia. Appl Neuropsychol. 2007;14(3):208-214.

14. Del Duca GF, Silva MC, Hallal PC. Incapacidade functional para atividades básicas e instrumentais da vida diária em idosos. Rev Saúde Pública. 2009;43(5):796-805.

15. Dias EG, Duarte YAO, Almeida MHM, et al. Caracterização das atividades avançadas de vida diária (AAVDS): um estudo de revisão. Rev Ter Ocup Univ São Paulo. 2011;22(1):45-51.

16. Al Snih S, Graham JE, Ray LA, et al. Frailty and Incidence of activities of daily living disability among older Mexican Americans. J Rehabil Med. 2009 Nov;41(11):892-7. doi: 10.2340/165019770424.

17. Walston J, Hadley EC, Ferrucci L, et al. Research agenda for frailty in older adults: toward a better understanding of physiology and etiology: summary from the American Geriatrics Society/ National Institute on Aging Research Conference on frailty in older adults. J Am Geriatr Soc. 2006;54:991-1001.

18. Folstein MF, Folstein SE, McHugh PR. "Mini-mental state": a practical method for grading the cognitive status of patients for the clinician. J Psychiatr Res. 1975;12(3):189-98.

19. Yu R, Morley JE, Kwok T, et al. The Effects of Combinations of Cognitive Impairment and Pre-frailty on Adverse Outcomes from a Prospective Community-Based Cohort Study of Older Chinese People. Front Med. 2018;5:50.

20. Tyrovolas S, Escriva NG, Ayuso-Mateos JL, et al. Frailty and health status of older individuals in three European countries: The COURAGE cross-sectional study. Exp Gerontol. 2018;106:137144. doi: 10.1016/j.exger.2018.02.028. Epub 2018 Feb 27.

21. Brigola AG, Rossetti ES, Dos Santos BR, et al. Relationship between cognition and frailty in elderly: a systematic review. Dement Neuropsychol. 2015 Apr-Jun;9(2):110-119. doi: 10.1590/1980-57642015DN92000005.

22. Lourenço RA, Moreira VLG, Banhato EFC, et al. Prevalence of frailty and associated factors in a community-dwelling older people cohort living in Juiz de Fora, Minas Gerais, Brazil: Fibra-JF Study. Ciênc Saúde Coletiva. 2019;24(1):35-44.

23. Brucki SM, Nitrini R, Caramelli P, et al. Sugestões para o uso do mini exame do estado mental no Brasil. Arq Neuropsiquiatr. 2003 Sep;61(3B):777-81. Epub 2003 Oct 28.

24. Katz S, Downs TD, Cares HR, et al. Progress in the development of the Index of ADL. Gerontologist. 1970 Spring;10(1):20-30.

25. Schneider RH, Marcolin D, Dalacorte RR. Avaliação funcional de idosos. Scientia Medica, Porto Alegre. 2008;18(1):4-9.

26. Lawton MP, Brody EM. Assessment of older people: self-maintaining and instrumental activities of daily living. Gerontologist. 1969;9:179-86.

27. Baltes PB, Mayer KU. The Berlin Aging Study: Aging from 70 to 100. New York: Cambridge University Press. 1999. Disponível em: http://www.base-berlin.mpg.de

28. Taylor HL, Jacobs DR Jr, Schucker B, et al. A questionnaire for the assessment of leisure time physical activities. J Chronic Dis. 1978;64:741-55.

29. Radloff LS. The CES-D scale: a self-report depression scale for research in the general population. Appl Psychol Meas. 1977;1:385-401.

30. Vella AR. Increasing use of cognitive measures in the operational definition of frailty-A systematic review. Ageing Res Rev. 2018(7);43:10-16.

31. Bunce D, Batterham PJ, Mackinnon AJ. Long-term Associations Between Physical Frailty and Performance in Specific Cognitive Domains. J Gerontol B Psychol Sci Soc Sci. 2018 Feb 1. doi: 10.1093/geronb/gbx177. [Epub ahead of print]

32. Lee $Y$. The effects of frailty and cognitive impairment on 3-year mortality in older adults. Maturitas. 2018;107:50-55.

33. Njegovan V, Hing MM, Mitchell SL, et al. The hierarchy of functional loss associated with cognitive decline in older persons. J Gerontol A Biol Sci Med Sci. 2001;56(10):M638-43.

34. Figueiredo CS, Assis MG, Silva LA, et al. Functional and cognitive changes in community-dwelling elderly: longitudinal study. Braz J Phys Ther. 2013 May-Jun;17(3):297-306.

35. Paterson $\mathrm{DH}$, Warburton DE. Physical activity and functional limitations in older adults: a systematic review related to Canada's physical activity guidelines. Int J Behav Nutr Phys Act. 2010 May 11;7:38. doi: 10.1186/1479-5868-7-38.

36. Christelis D, Dobrescu LI. The impact of social activities on cognitive ageing: evidence from eleven European countries. Centre for Studies in Economics and Finance Working Papers 320 Nápoles:Centre for Studies in Economics and Finance (CSEF), Universidade de Nápoles; 2012.

37. Ávila-Funes JA, Melano-Carranza E, Payette $\mathrm{H}$, et al. Síntomas depressivos como fator de riesgo de dependência en adultos mayores. Salud Publica Mex. 2007 Sep-Oct;49(5):367-75. 